\title{
Channel State Information Estimation In MIMO-OFDM Wireless Systems
}

\author{
Hardeep Singh ${ }^{1}$, Ishan Khurana ${ }^{2}$ \\ ${ }^{I}$ Department of Electronics and Communication Engineering, Lovely Professional University, India \\ ${ }^{2}$ Department of Electronics and Communication Engineering, Lovely Professional University, India
}

\begin{abstract}
Multiple Input Multiple Output (MIMO) in combination with Orthogonal Frequency Division Multiplexing (OFDM) can provide spectrally efficient and ISI free communication. Channel estimation is of great importance in order to recover the signal at the receiver side. Therefore accurate channel state information is essential for proper detection and decoding in MIMO-OFDM wireless systems. To estimate channel state information various types of techniques are being deployed in these systems. Accuracy and precision of channel estimation depends on the techniques used for the purpose of estimating channel state information. The more the accuracy of the technique, more will be the accurate performance of the system. In this paper we provide a review of the various types of techniques that are used in estimation of channel in MIMO-OFDM systems. Although the existing CE techniques for OFDM can be employed for MIMO-OFDM systems too but the computation complexity is increased in the latter. The techniques can be chosen on the basis of their performance which can be judged by Mean Square Error (MSE), convergence speed, and the stability of the algorithm. Various CE techniques like LS and MMSE can be used. Moreover adaptive CE techniques like LMS, NLMS, RLS are gaining more attention. These can be chosen based on the system requirement and the environment in which the system is to be deployed. Comparison of the techniques on the basis of above given parameters is shown in this paper. Also the improvement aspects for techniques of channel estimation are discussed.
\end{abstract}

Keywords:- Multiple Input Multiple Output systems(MIMO), Channel Estimation(CE), LMS, MMSE, NLMS, $R L S$

\section{Introduction}

Orthogonal frequency division multiplexing (OFDM) has been accepted as a promising air -interface due to its high spectrum efficiency. High spectrum efficiency is provided due to the fact that in this whole spectrum is shared by all the OFDM sub carriers that are orthogonal to each other. FFT and IFFT operations are used in OFDM due to which the oscillators are not required at the transmitter and receiver side. Thus reduces the complexity at transmitter and receiver and also they are fast algorithms for implementing DFT and IDFT which decreases the computation complexity as compared to DFT and IDFT. Moreover it provides ISI free communication due to the use of $\mathrm{CP}$ (cyclic prefix) which is just the repetition of tail of the symbol at the front part of the symbol. OFDM acts as a standard for many wireless applications like Digital Video Broadcasting (DVB), Digital Audio Broadcasting (DAB) [1] [2], WIMAX, Wireless Local Area Network (WLAN) and ADSLs [1] [5].

If multiple transmit and receive antennas are used then the capacity of the system can be increased. The systems which use multiple antennas at the transmitter and receiver are called MIMO systems [3]. The capacity of the MIMO system can be improved by a factor equal to minimum number of antennas employed at the transmitter and receiver. Transmission rate is increased in case of spatial multiplexing while BER enactment is improved in case of spatial diversity. Therefore, these are widely used in many wireless applications in combination with OFDM forming MIMO-OFDM system. Parallel transmission is done by dividing whole channel into many sub-channels, thus attaining high data rate and increasing symbol duration to battle ISI. STBCs are used to increase the diversity gain in MIMO systems. Channel capacity and multiplexing gain is increased by spatial multiplexing (SM) [5].The challenging problem for wireless systems is channel estimation. In wireless systems channels are dynamic in nature as compared to guided media. The signal is received at the receiver after undergoing many adverse effects due to reflection, scattering and diffraction and that too from multipath. Channel response is time variant due to mobility of transmitters, receivers and other obstacles. The signal spreads over the statistics like frequency, time, phase. These statistics define the channel selectivity and has a great impact on received signal. These effects of the channel on its response has to be known which is known as channel estimation or channel state information estimation. For data detection and equalization we need channel State Information (CSI) at the receiver side. Broadly if we classify channel estimation then there are two ways for channel estimation- one is the Training based channel estimation and second one is blind channel estimation. There is also one more of its type called semi blind channel estimation because it employ 
both of the techniques. It the combination of the above two. Training based channel estimation uses two types of pilot types i.e. block type and comb type [2]. In comb type the pilots are inserted into certain sub-carriers of each OFDM symbol and not in all the subcarriers while in case of block type the pilots are inserted into all subcarriers of OFDM symbol within some predefined period. Also comb type is mostly used for fast fading channels while the bock type is used for slow fading channels. Comb type pilot organization outperforms block type pilot organization. Other type is the blind channel estimation which exploits the statistical facts of the symbols that are received at the receiver. But this type of channel estimation can only be used for slow time varying channels. Moreover this type of channel estimation technique increases the complexity at the receiver. Although pilot based channel estimation (CE) consumes bandwidth more than blind type but it is a good candidate for fast time varying channels [5]. CE methods used are Least Square (LS) [9] and Minimum Mean Square Error (MMSE) [9]. In addition to this adaptive CE algorithms are gaining more attention these days. Least Mean Square (LMS) [10] is widely used for its simplicity. Improved version of standard LMS i.e. Normalized Least Mean Square (NLMS) [12] is also been used. If complexity is not an issue then Recursive Least Square (RLS) [10] [11] is a good choice. Moreover to use the best part of the above given Adaptive Channel Estimation (ACE) algorithms they can be combined to build the hybrid algorithms. Leaky Least Mean Square (LLMS) [13] algorithm is such an algorithm.

We will use the following notations throughout the paper $-(.)^{*}$ represents the conjugate complex of the vector or variable, E(.) represents the expectation operator and $(.)^{\mathrm{H}}$ represents the hermitian or we can say conjugate transpose. All the variables used are vectors as here we are dealing with MIMO systems therefore inputs and outputs are not scalars rather they are vectors.

Further the next sections are organized as follows. The section II depicts the MIMO-OFDM system and the system model. CE methods are described in section III. Section IV discusses the adaptive CE algorithms with appropriate equations. Finally Section V concludes the paper and it is acknowledged in section VI.

\section{MIMO-OFDM System}

MIMO in combination with OFDM is widely used now-a-days due its best performance in terms of capacity of channels, high data rate and good outcome in frequency selective fading channels. In addition to this it also improves reliability of link. So it is widely used in future broadband wireless system/communications. Cyclic prefix is the copy of last part of OFDM symbol which is appended to that OFDM symbol that is to be transmitted. It is basically $0.25 \%$ of the OFDM symbol. We can say that one fourth of the OFDM symbol is taken as CP (cyclic prefix) and appended to each OFDM symbol. IFFT is used at the transmitter and FFT is used at the receiver which substitutes the modulators and demodulators. Doing so eliminates the use of banks of oscillators and coherent demodulators [4]. Moreover the complex data cannot be transmitted as it is therefore it is first converted to analog form which is accomplished by IFFT. It basically converts the signal from frequency domain to time domain. If we assume $\mathrm{X}[\mathrm{i}]$ is the signal which is input to the IFFT block and $\mathrm{N}$ is the channel number then output $\mathrm{X}[\mathrm{n}]$ is given by:

$$
\begin{gathered}
\operatorname{IFFT}\{X[i]\}=X[n]=\frac{1}{N} \sum_{n=0}^{N-1} X[i] e^{\frac{j 2 \pi n i}{N}} \\
\text { Where, } 0 \leq n \leq N-1
\end{gathered}
$$

Prior to IFFT operation symbol mapping is performed which is nothing but the modulation block. Any of the widely used modulation techniques can be applied like BPSK, QPSK, QAM, PSK etc. Further there are higher order modulations are also available which provide more capacity at little expense of BER performance degradation. After IFFT block pilot insertion is done and then CP (cyclic prefix) is added.

Figure 1 below shows the block diagram constituting MIMO and OFDM. Any antenna configuration for the MIMO can be used according to the system requirement. Higher the configuration more will be the capacity and more will be the computational complexity of the transceiver design. If we see the case of estimating channel then also the computational complexity is increased. Mapper defines the modulation to be used. Symbol encoder takes the shape of the STBC (Space Time Block Code) if spatial diversity is to be used and it takes the shape of the demultiplexer/multiplexer if spatial multiplexing is to be used. In SISO (Single Input Single Output) only single antenna is used both at the transmitter and the receiver. Due to this reason the data transfer rate is not very fast. Also if we see it in case of reliability of data then also it is not good. As diversity is not achieved which is the back bone for reliable communication. If the data is lost then it cannot be recovered back as no replicas are sent, only original signal is sent on the single antenna. Moreover in SISO the implementation of time and frequency diversity can only be done. There are other forms also like SIMO (Single Input Multiple Output) in which transmitting antenna used is only one while receiving antennas used are multiple and MISO (Multiple Input Single Output) in which receiving antenna is single while multiple antennas are used at the transmitter side. In SIMO spatial diversity can be achieved as multiple antennas are used at the receiving side only but spatial multiplexing cannot be achieved while in case of MISO spatial diversity cannot be achieved but spatial multiplexing can be achieved as multiple number of antennas are used at the transmitting 
side. All these properties or we can say the pros of both these systems i.e. SIMO and MISO are combined to a single form known as MIMO which we have introduced earlier.

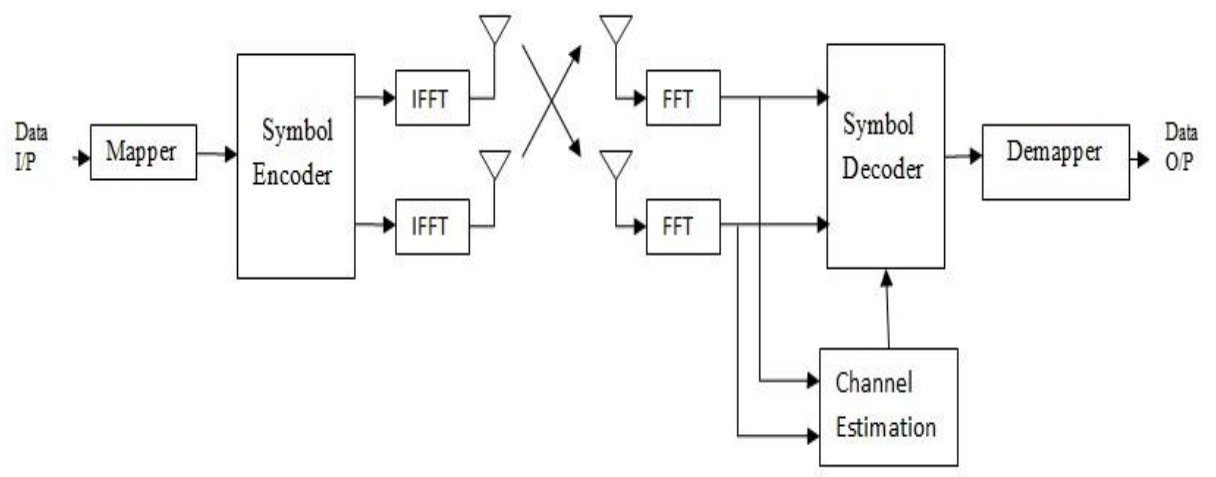

Figure 1. MIMO-OFDM system model.

The MIMO-OFDM system model [4] with NR receive antennas and NT transmit antennas can be given as:

$$
\left[\begin{array}{c}
Z_{1} \\
Z_{2} \\
\vdots \\
Z_{N}
\end{array}\right]=\left[\begin{array}{cccc}
H_{1,1} & H_{1,2} & \ldots & H_{1, N T} \\
H_{2,1} & H_{2,2} & \ldots & H_{2, N T} \\
\vdots & \vdots & \ddots & \vdots \\
H_{N R, 1} & H_{N R, 2} & \ldots & H_{N R, N T}
\end{array}\right]\left[\begin{array}{c}
A_{1} \\
A_{2} \\
\vdots \\
A_{N T}
\end{array}\right]+\left[\begin{array}{c}
M_{1} \\
M_{2} \\
\vdots \\
M_{N T}
\end{array}\right]
$$

Where, $\mathrm{Z}$ represents $\mathrm{O} / \mathrm{P}$ data vector, $\mathrm{H}$ denotes Channel matrix, A denotes I/P data vector and $\mathrm{M}$ represents Noise vector.

The wireless channel may be of any type like we can say it can be AWGN, Rayleigh or Rician depending upon the LOS (line of sight) component present or absent. For the latter it is present while it is absent in the former. After receiving the signal the $\mathrm{CP}$ is removed then the pilots are also removed from main signal received. After this the signal that is in time domain can be again converted to frequency domain by taking FFT of the received signal. If we assume $\mathrm{X}[\mathrm{n}]$ is the received signal then its FFT $\mathrm{X}[\mathrm{i}]$ can be given as:

$$
\operatorname{FFT}\{X[n]\}=\mathrm{X}[\mathrm{i}]=\sum_{\mathrm{i}=0}^{\mathrm{N}-1} \mathrm{X}[\mathrm{n}] \mathrm{e}^{\frac{-\mathrm{j} 2 \pi \mathrm{ni}}{\mathrm{N}}}
$$

The sequence on each of the OFDM block is then provided to channel estimation block where the received pilots altered by channel are compared with the original sent pilots. Channel estimation block consists of the algorithms that are applied to estimate the channel. These are discussed below in the following sections.

\section{CE Methods}

CE (channel estimation) methods are divided into two types. One is training based and the other one is blind i.e. without training sequences. There are various types of channel estimations and broadly they can be classified as Training based estimation, semi blind estimation and blind channel estimation. Training based requires pilot bits to be sent along with the data. Arrangement of pilot bits can be block type [6] and comb type [7]. In block type transmission of pilot is done on each and every subcarrier at successive intervals of time. While in comb type pilots are sent for whole time i.e pilots are implanted into apiece OFDM emblem. Blind channel approximation is done by exploiting the statistical [7] properties of the network. It is advantageous to use as it does not wastes bandwidth as no pilots are needed. But it has performance less than pilot based so rarely used. Moreover it makes the receiver more complex. Let us consider the simplified received equation as:

$$
\mathrm{Z}=\mathrm{HA}+\mathrm{M}
$$

Where $\mathrm{O}$ is the received vector, $\mathrm{H}$ channel matrix, $\mathrm{A}$ is the input data matrix and $\mathrm{M}$ is the noise matrix.

\section{A. LS CE method}

The Least Square (LS) estimator is the Least Square estimator is used for estimating the channel by minimizing the squared error between estimated value and the desired value or we can say desired value or actual values we got earlier. It is given by:

$$
\hat{E}_{L S}^{\prime}=H^{-1} A
$$

Where $\mathrm{H}$ represents the channel matrix and $\mathrm{A}$ is the input matrix.

B. MMSE CE method 
The Minimum Mean Square Error (MMSE) estimator computes the mean square error then minimizes the same. If we see the performance wise this estimator performs better than the previously discussed LS type. But it has one con that it increases the complexity in terms of computations. Here $(.)^{\mathrm{H}}$ represents hermitian (conjugate transpose). MMSE is given by:

$$
H_{M M S E}^{\wedge}=R_{A Z} R_{Z Z}^{-1} Z
$$

Where $R_{A Z}$ is cross-covariance of $A \& Z$ and $R_{Z Z}$ is auto-covariance of $Z$

Also,

$$
\begin{aligned}
R_{\theta Z}=E\left[H Z^{H}\right]=E[\theta(H \theta & \left.+M)^{\mathrm{H}}\right]=R_{A A} H^{H} \\
\& R_{Z Z}=E\left[Z Z^{H}\right] & =E\left[(H A+M)(H A+M)^{\mathrm{H}}\right] \\
& =E\left[H A(H A)^{\mathrm{H}}+H A M^{H}+M(H A)^{\mathrm{H}}+M M^{H}\right] \\
& =H R_{A A} H^{H}+\sigma_{n}^{2} I
\end{aligned}
$$

Here $R_{A A}=E\left[A A^{H}\right]$ which denotes the auto covariance of channel matrix $A, \sigma_{n}^{2}$ is noise variance and I is the identity matrix. Noise variance is given as $\sigma_{n}^{2}=E\left[M^{2}\right] . R_{\theta \theta} \& \sigma_{n}^{2}$ are known to the estimator a priori.

Now put values in (6) we get, $\theta_{M M S E}^{\prime}=R_{A A} S^{H}\left(H R_{A A} H^{H}+\sigma_{n}^{2} I\right)^{-1} \mathrm{Z}$

$$
\theta_{M M S E}^{\prime}=R_{A A}\left(R_{A A}+\sigma_{n}^{2}\left(H^{H} H\right)^{-1}\right)^{-1} H^{-1} Z
$$

\section{Adaptive CE Methods}

Adaptive CE (channel estimation) methods or algorithms are being widely deployed in channel estimation. As we know that the wireless channel is time varying and totally random in nature. Therefore to keep track of it an adaptive algorithm best suits it. These CE algorithms after successive iterations converges to the optimum solution [8]. Also they provide good tracking capability. Various adaptive CE estimators available are LMS, RLS, NLMS etc. They continuously update their parameters until they reach the optimum solution. Moreover they need only the received signal which includes the training sequences which were sent at the transmitter. These are known to the receiver which are used by these adaptive CE algorithms to check the error value or we can say that to minimize the error value in order to reach the optimum solution. Updating the parameters is dependent on the step size parameter in case of stochastic gradient algorithms [8]. The greater the step size the more will be the convergence speed. The time required by the algorithm to reach the optimum solution decreases hence the steady state error is reached. While if it increases too much then there is a chance that system may become unstable. If the case of recursive algorithms is seen we see that they are not dependent on the step size parameter, thus making them good and fast estimators. But there is a con in them i.e. they are very complex. Their complex structure requires more hardware cost also. Though they are faster than stochastic gradient algorithm but complexity marks them as unusable but now the scenario is changing with the improved hardware structures in use.

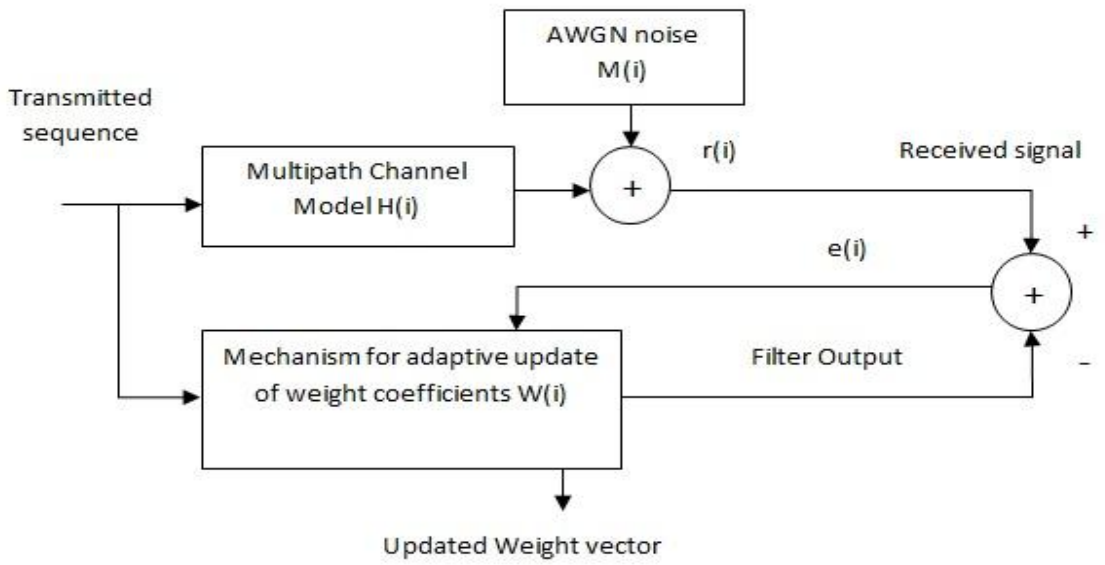

Figure 2 Adaptive CE method

\section{A. LMS ACE method}

Least Mean Square (LMS) method or algorithm is widely used in numerous applications which includes system identification. i.e. it is an adaptive channel estimation technique. LMS is a very simple and adaptive CE method among others. LMS has slow convergence speed. Moreover its complexity is less. Basically LMS algorithm can be expressed as follows:

$$
w(i+1)=w(i)+u x(i) e(i)^{*}
$$


Where, $w(i)$ is initial weight vector, $w(i+1)$ is final weight vector $u$ is step size, $x(i)$ is input vector and $e(i)$ is error signal. Also $0<u<\frac{2}{\lambda_{\max }}$ [8]. $\lambda_{\max }$ is the maximum eigen value of the correlation matrix. And error signal is expressed as:

$$
e(i)=d(i)-y(i)
$$

Where $d(i)$ is the desired value and $y(i)$ is the filtered output or estimated value which can be expressed as:

$$
y(i)=w(i)^{H} x(i)
$$

As the value of $u$ is increased ,convergence speed is increased but the performance is degraded as less data is taken for processing. Value of $\mathrm{u}$ has to be adjusted according to the environment. For fast changing environment u should be as small as possible while for slow varying environment it should be kept large enough to reduce error probability. Its performance is robust.

\section{B. NLMS ACE method}

Normalized Least Mean Square (NLMS) method or algorithm is variant of LMS algorithm. In standard LMS the correction term is directly proportional to $\mathrm{x}(\mathrm{i})$. as it increases the gradient noise increases. So as to reduce this the correction term [8] is normalized by dividing it by Euclidean norm of x(i). It is widely used in numerous applications which includes system identification. i.e. it is an adaptive channel estimation technique. NLMS algorithm can be expressed as follows:

$$
w(i+1)=w(i)+\frac{u x(i) e(i)^{*}}{\|x(i)\|^{2}}
$$

Where, $w(i)$ is initial weight vector, $w(i+1)$ is final weight vector $u$ is step size, $x(i)$ is input vector and $e(i)$ is error signal. And error signal is expressed as:

$$
e(i)=d(i)-y(i)
$$

Where $d(i)$ is the desired value and $y(i)$ is the filtered output or estimated value which can be expressed as:

$$
y(i)=w(i)^{H} x(i)
$$

NLMS can be viewed as LMS having time varying step size $u$. its convergence speed is faster than LMS. Normalization increases its speed and also decreases the error floor.

\section{RLS ACE method}

The Recursive Least Square (RLS) method as its name suggest i.e. recursive LS, means that LS is used recursively. Here the past or previously calculated estimates are used to find the new estimates. The input values and the output values which are desired are calculated are present at each iteration [11]. The algorithm can be expressed as follows:

$$
w(i)=w(i-1)+D(i) e(i)^{*}
$$

Where $e(i)$ is error signal which can be expressed as:

$$
e(i)=d(i)-x(i) w(i-1)^{H}
$$

Where $d(i)$ is desired value, $x(i)$ is input value and $x(i) w(i-1)^{H}$ is the estimated value. Also,

$$
D(i)=P(i) x(i)
$$

Where $P(i)=\theta(i)^{-1}$ and is given as:

$$
\theta(i)^{-1}=\lambda^{-1} \theta^{-1}(i-1)-\frac{\lambda^{-2} \theta^{-1}(i-1) x(i) x^{H}(i) \theta^{-1}(i-1)}{1+\lambda^{-1} x^{H}(i) \theta^{-1}(i-1) x(i)}
$$

Here $\theta(i)^{-1}$ is the correlation matrix 
$\lambda$ is the forgetting factor. It weights recent data more heavily. It does not affect rate of convergence and it determines tracking capability. Smaller its value better would be the performance. If it is too large then algorithm becomes unstable. RLS based estimation of channel is said to have very good performance than LMS algorithm but the computation complexity of the algorithm is large as compare to LMS. It provides high rate of convergence.

\section{LLMS ACE method}

LLMS (Leaky Least Mean Square) algorithm is the combination of two LMS algorithms. The error signal of one LMS algorithm is fed back to the other LMS algorithm. By doing so the weight vectors are updated twice i.e. by the first LMS and then by the second LMS. The error signal is combined thus making it as the efficient estimator increasing the convergence speed and providing lower error floor than the single LMS being used in the system. LMS1 Output is multiplied by image array factor $\left(A^{\prime}\right)$ of desired signal. The resultant output is then provided to LMS2 for further processing. Error signal from LMS2 is fed back to LMS1. d1 and $\mathrm{d} 2$ external reference signal is provided to them respectively. The error signal at $i^{\text {th }}$ iteration is given by:

$$
\begin{gathered}
e_{L L M S}(i)=e_{1}(i)-e_{2}(i-1) \\
e_{1}(i)=d_{1}(i)-w_{1}^{H}(i) x_{1}(i) \\
e_{2}(i)=d_{2}(i)-w_{2}^{H}(i) x_{2}(i)
\end{gathered}
$$

The final updating equation for LLMS can be expressed as:

$$
w_{j}(i+1)=w_{j}(i)+u_{j} e_{j}(i) x_{j}(i)
$$

Where $0<u_{i}<u_{0}$ and $\mathrm{j}=1$ for LMS1 and 2 for LMS2. $\mathrm{U}$ is step size parameter and $u_{0}$ is positive number, depends on signal statistics.

\section{Conclusion}

Estimation of the channel coefficients is a challenging task in MIMO-OFDM systems. Moreover it complex task than in simple OFDM systems. In this paper we presented the techniques used for channel state information estimation in MIMO-OFDM systems. The techniques discussed above are based on training sequence based channel estimation. While observing the CE algorithms we conclude that MMSE algorithm outperforms LS algorithm. But the former has a disadvantage as it is more complex than LS. So LS is to be preferred if complexity is not desirable at the receiver while MMSE is to be preferred if complexity is not an issue. These are the simplest estimators and various other variants are also available like Low Rank Minimum Mean Square Error (LMMSE) which is a low rank MMSE. Though performance is similar to MMSE but complexity is reduced to great extent.

Next we described the adaptive CE algorithms. Due to their adaptive nature they are very good at tracking the time varying wireless channel in MIMO-OFDM systems. LMS is the simplest among the discussed adaptive CE methods. Complexity of LMS is low therefore it is preferred in channel estimation. It has drawback that its convergence speed is low. Moreover it is dependent on step size parameter. NLMS on the other hand is complex than LMS but its convergence speed is more than LMS. Its error floor is lower than standard LMS. So BER performance of NLMS is better than LMS. Coming to the RLS algorithm, it is more complex than others. But it provides better performance than LMS algorithm. In terms of reaching the optimum solution i.e. its convergence speed is more than the rest. To reduce error floor i.e. increasing BER performance and robustness hybrid algorithms can be made by combination of these algorithms. LLMS has also been used in channel estimation and its performance is improved than single LMS. As a future work other combinations may be tested for their performance in channel estimation for MIMO-OFDM systems in wireless systems.

\section{References}

[1] IEEE Computer Society LAN MAN Standards Committee. "IEEE 802.11: Wireless LAN medium access control and physical layer specifications.", 1999.

[2] Wang, Han, and JinKuan Wang, "Optimal pilot design for MIMO-OFDM system channel estimation in time domain." IEEE IWSDA 3rd International Workshop on Signal Design and Its Applications in Communications, pp. 307-312, 2007.

[3] Hwang, Taewon, et al. "OFDM and its wireless applications: a survey." IEEE Transactions on Vehicular Technology, vol. 58,no. 4, pp. 1673-1694, 2009.

[4] Shin, Myeongchoel, Hakju Lee, and Chungyong Lee, "Enhanced channel-estimation technique for MIMO-OFDM systems." IEEE Transactions on Vehicular Technology, vol. 53, no. 1, pp. 261-265, 2004.

[5] Hidayat, Risanuri, Anggun Fitrian Isnawati, and Budi Setiyanto, "Channel estimation in MIMO-OFDM spatial multiplexing using Least Square method." IEEE International Symposium on Intelligent Signal Processing and Communications Systems (ISPACS), pp. $1-5,2011$.

[6] Coleri, Sinem, Mustafa Ergen, Anuj Puri, and Ahmad Bahai "Channel estimation techniques based on pilot arrangement in OFDM systems", IEEE Transactions on Broadcasting, vol. 48, no. 3, pp. 223-229, 2002. 
[7] Bagadi, Kala Praveen, and Susmita Das, "MIMO-OFDM channel estimation using pilot carries", International Journal of Computer Applications, vol. 2, no. 3, pp. 81-88, 2010.

[8] Haykin, Simon S., "Adaptive Filter Theory", Pearson Education, $3^{\text {rd }}$ edition, 2002.

[9] Krouma, Houda, Malek Benslama, and Farouk Othmani-Marabout, "Low rank MMSE channel estimation in MIMO-OFDM systems." IEEE Second International Conference on Innovative Computing Technology (INTECH), pp. 279-284, 2012.

[10] Rana, Md Masud, "Performance comparison of LMS and RLS channel estimation algorithms for 4G MIMO OFDM systems." IEEE 14th International Conference on Computer and Information Technology (ICCIT), pp. 635-639, 2011.

[11] Rana, Md Masud, and Md Kamal Hosain, "Adaptive Channel Estimation Techniques for MIMO OFDM Systems." International Journal of Advanced Computer Science and Applications, vol. 1, no. 6, pp. 134-138, 2010.

[12] Arslan, H. U. S. E. Y. I. N. "Channel estimation for wireless OFDM systems.", IEEE Surveys and Tutorials, vol. 9, no. 2, pp. 1848, 2007.

[13] Bhoyar, Dinesh B., C. G. Dethe, M. M. Mushrif, and Abhishek P. Narkhede, "Leaky Least Mean Square (LLMS) Algorithm For Channel Estimation In BPSK-QPSK-PSK MIMO-OFDM System", IEEE International Multi-Conference on Automation, Computing, Communication, Control and Compressed Sensing, pp. 623-629, 2013 